\title{
La maladie de Berger : une néphropathie à IgA
}

La maladie de Berger à IgA est une des glomérulonéphrites les plus communes chez l'homme. Caractérisée par des dépôts d'IgA dans le mésangium glomérulaire, elle progresse souvent vers l'insuffisance rénale sévère avec la sclérose progressive des capillaires glomérulaires. Les causes et mécanismes de cette affection restent incertains. Une prédisposition génétique est probable et la présence chez les malades d'IgA circulantes anormales pourrait constituer une piste éthiopathogénique intéressante.

Philippe Lesavre Laure-Hélène Noèl Renato Monteiro Lise Halbwachs-Mecarelli

\section{ADRESSE}

Ph. Lesavre: professeur de néphrologie. L.H. Noël: chargée de recherche à l'Inserm. R. Monteiro: chargé de recherche à l'Inserm. L. Halbwachs-Mecarelli: chargée de recherche au Cnrs. Hôpital Necker, Inserm U. 25, 161, rue de Sèvres, 75743 Paris Cedex 15, France. a maladie de Berger (ou néphropathie à $\operatorname{Ig} A$ ) est la plus fréquente des maladies glomérulaires dans de nombreux pays. Sa première définition par Berger et Hinglais, en 1968, reste aujourd'hui totalement valide: glomérulonéphrite caractérisée par la présence en immunofluorescence de dépôts mésangiaux d'IgA prédominants ou exclusifs et par l'absence de signes extrarénaux [1]. L'origine des IgA mésangiales et le mécanisme de leur dépôt restent en grande partie inconnues. Cette méconnaissance est grave puisqu'il s'agit d'une maladie fréquente et sévère. Elle est en effet la cause principale, environ un quart des cas, des glomérulonéphrites humaines primitives. Cette fréquence est à peu près identique dans le monde entier; elle est cependant influencée par des facteurs ethniques ou géographiques, puisqu'elle est exceptionnelle chez les Noirs des États-Unis et d'Afrique du Sud, par exemple. Elle touche essentiellement des enfants et des adultes jeunes et deux fois plus souvent l'homme que la femme. Sa gravité est grande puis- que, dans $25 \%$ des cas, elle évolue vers l'insuffisance rénale terminale. Elle est responsable d'environ $10 \%$ des cas d'insuffisance rénale terminale, soit 200 à 300 nouveaux malades hémodialysés ou transplantés annuellement en France. Cette gravité ainsi que l'absence de traitement de la maladie expliquent l'effort de recherche entrepris par de nombreuses équipes.

\section{Expression clinique et anatomo-pathologique}

Compte tenu de sa définition, le diagnostic de maladie de Berger repose entièrement sur l'examen histologique du tissu rénal. La maladie est en effet définie par la présence, démontrée par l'examen en immunofluorescence, d'immunoglobulines principalement (ou exclusivement) de classe $\operatorname{IgA}$, dans le mésangium* de tous les glomérules (figure 1 ). Ces dépôts ont une distribution intercellulaire, analogue à celle observée

\footnotetext{
* Mésangium: tissu intercapillaire, séparant, dans le glomérule rénal, les différentes branches du bouquet de vaisseaux capillaires.
} 
chez l'animal après injection de complexe antigène-anticorps ou d'IgG agrégées [2]. Les espaces intercellulaires mésangiaux sont dans une situation très particulière puisqu'une substance ou une particule peuvent y pénétrer directement à travers les fenestrations endothéliales, sans avoir à franchir la membrane basale capillaire. Dans la maladie de Berger, les dépôts d'IgA restent extracellulaires et ne sont pas phagocytés par les cellules mésangiales (figure 2). Ils sont très souvent associés à des dépôts de $\mathrm{C} 3$, alors que les protéines de la voie classique du complément $(\mathrm{Cl}$ et $\mathrm{C} 4)$ sont absentes. Dans un tiers des cas environ, les dépôts contiennent aussi des IgG [3] et plus rarement des IgM. Enfin, le complexe lytique du complément (C5b-C9) est présent dans la majorité des cas [4], parallèlement au C3 et aux IgA. Son rôle lésionnel est probable, mais des lésions tissulaires

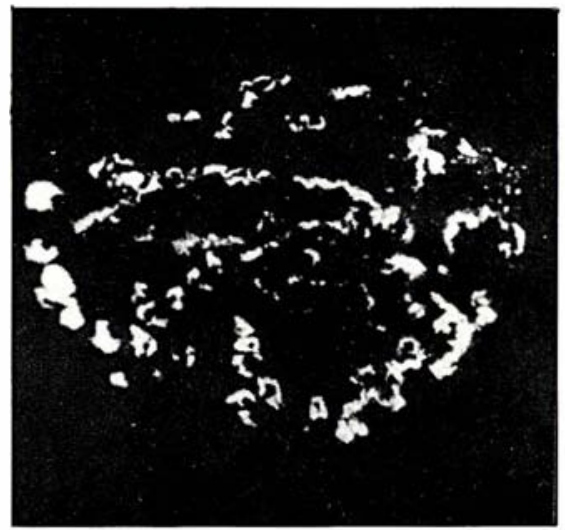

Figure 1. Dépôts mésangiaux d'IgA révélés par l'étude en immunofluorescence directe $(\times 250)$ à l'aide d'anticorps anti-chaine $a$ humaine fluorescents /cliché J. Berger). sont présentes dans certains cas en l'absence de dépôts de complément. En microscopie optique, les aspects lésionnels sont très variables. Lorsqu'existent des lésions glomérulaires, elles sont constituées par une hypertrophie de la matrice et, parfois une augmentation de la cellularité mésangiales. Très fréquemment s'y ajoutent des lésions dites «segmentaires et focales ». Ces lésions associent une thrombose capillaire puis une nécrose et une prolifération segmentaire des cellules épithéliales. Elles évoluent finalement vers une lésion irréversible de sclérose de l'anse des capillaires glomérulaires. La diffusion de ces lésions évolutives marque les formes graves ; l'apparition d'une insuffisance rénale dépend de leur éiendue, d'autant qu'elles s'associent de façon parallèle à des lésions cubulaires et interstitielles rénales [5].

La maladie peut survenir à tous les

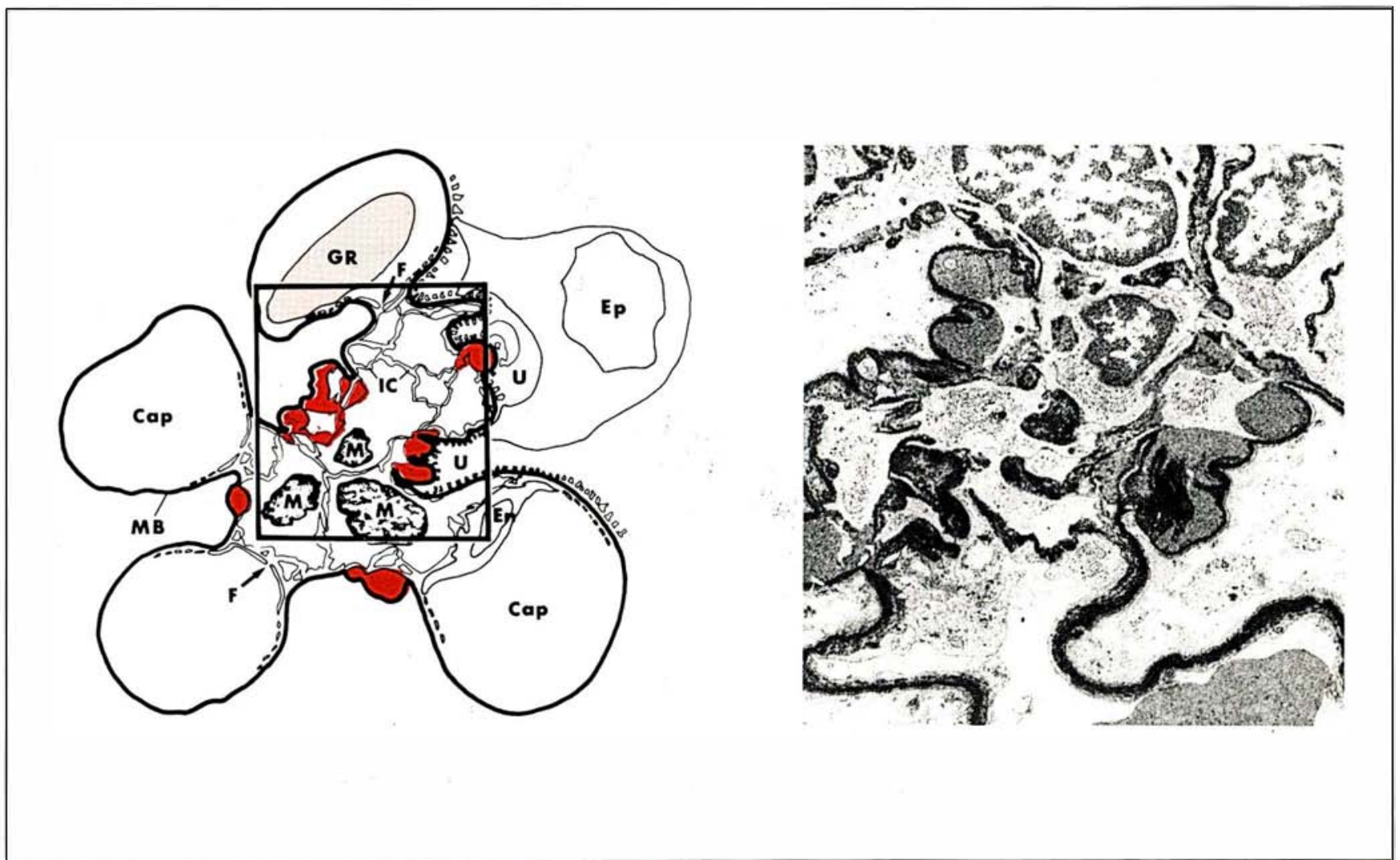

Figure 2. Tige mésangiale comportant des dépôts électroniquement denses. A droite: aspect en microscopie électronique (cliché $\mathbf{N}$. Hinglais) (imprégnation à l'argent marquant les membranes basales glomérulaires et le tissu intercellulaire, $X 15$ 000). A gauche: représentation schématique des dépôts d'lgA. Ils sont situés dans les espaces intercellulaires mésangiaux (IC) le long de la membrane basale glomérulaire (MB) et surtout en regard de l'espace urinaire (U). II n'existe pas d'infiltration cellulaire anormale. $(G R=$ globule rouge; $M=$ cellule mésangiale; Ep = cellule épithéliale ; En = cellule endothéliale $; U=$ espace urinaire ; Cap = capillaire). 


\section{RÉFÉRENCES}

1. Berger J, Hingins N. Les dépôts intercapillaires d'IgA-IgG. J Urol Nephrol (Paris) 1968 ; $74: 694-5$

2. Michael AF. The glomerular mesangium. Kidney Int 1980 ; 17 : 141-7.

3. Aucouturier P, Monteiro RC, Noèl LH, Preud'homme JL, Lesavre P. Glomerular and serum immunoglobulin $\mathrm{G}$ subclasses in IgA nephropathy. Clin Immunol Inımunopathol 1989 (sous presse)

4. Rauterberg EW, Lieberknecht HM, Wingen AM, Ritz E. Complement membrane attack (MAC) in idiopathic IgAglomerulonephritis. Kidney Int 1987 ; 31 : 820-9.

5. Droz D, Kramar A, Nawar T, Noèl LH. Primary IgA nephropathy: pronostic factors. Contr Nephrol 1984 ; 40 : 202-7.

6. D'Amico G. Natural history and treatment of idiopathic IgA nephropathy. In : Robinson RA, ed. Nephrology. New York: Springer, $1984: 686-701$

7. Nicholls KM, Fairley KF, Dowling JP, Kincaid-Smith P. The clinical course of mesangial IgA-associated nephropathy in adults. $Q$ J Med 1984 ; 210 : 227-50.

8. Beukhof JR, Kardaun O, Schaafsma W. Toward individual prognosis of IgA nephropathy. Kidney Int 1986 ; 29 : 549-55.

9. Berger J, Noèl LH, Nabarra B. Recurrence of mesangial $\operatorname{IgA}$ nephropathy after renal transplantation. Contrib Nephrol 1984; 40 : 195-7.

10. Levy M. Do genetic factors play a role in Berger's disease? Pediatr Nephrol 1987; l: 447-54.

11. Simon F, Ang KS, Bavay P, et al. Glomérulonéphrite à $\operatorname{IgA}$. Épidémiologie dans une population de 250000 habitants. Presse Med 1984 ; 13 : 257-60.

12. Julian BA, Quiggins PA, Thompson JS, Woodford SY, Gleason K, Wyatt RJ. Familial $\operatorname{Ig} A$ nephropathy. Evidence of an inherited mechanism of disease. $N$ Engl J Med 1985 ;

âges de la vie, mais elle touche essentiellement l'adulte jeune et l'enfant. Deux types de signes révélateurs sont habituels : (1) hématurie microscopique et protéinurie asymptomatique ; (2) hématurie macroscopique : lorsqu'un cas, particulièrement chez l'adulte, a été révélé fortuitement par la découverte d'une protéinurie ou d'une hématurie microscopique, l'apparition d'une hématurie macroscopique ultérieure est rare. Réciproquement, les épisodes d'hématurie macroscopique sont révélateurs et fréquemment récidivants. Ils apparaissent souvent au cours d'un épisode infectieux banal des voies aériennes supérieures, plus rarement au cours d'un effort physique important. L'intervalle entre infection d'allure virale et épisode d'hématurie est trop court (24 à 48 heures) pour pouvoir évoquer la seule responsabilité de la réponse anticorps de classe $\operatorname{Ig} \mathrm{A}$ qui est plus tardive. Enfin l'absence de signes extrarénaux permet de distinguer la maladie de Berger de la glomérulonéphrite du purpura rhumatoïde, également caractérisée par des dépôts mésangiaux d'IgA, mais qui comporte en plus des signes de vascularité cutanée, digestive et articulaire, et dont l'évolution est aiguë (quelques semaines à quelques mois). Les rapports entre les deux maladies sont certains, puisqu'il existe des formes de passage lors de l'évolution chez un même malade et des cas où maladie de Berger et glomérulonéphrite du purpura rhumatoïde touchent deux membres d'une même famille.

L'hypertension artérielle complique la maladie selon une fréquence croissante avec l'âge du patient et la durée de l'évolution de la maladie. La sévérité de la sclérose et de la fibrose des artérioles rénales est assez bien corrélée à la présence de l'hypertension artérielle et à l'importance de la sclérose glomérulaire. La question du rôle lésionnel des dépôts d'IgA dans les vaisseaux extraglomérulaires est posée; il nous semble, pour notre part, que les dépôts sont limités au mésangium glomérulaire, rendant plus probable un mécanisme indirect, non immunologique, éventuellement hémodynamique, pour expliquer ces lésions. Ces lésions vasculaires jouent certainement un rôle dans l'apparition et la progres- sion de l'insuffisance rénale. L'évolution à long terme est plus sévère qu'elle ne le semblait dans les années qui ont suivi sa description, car l'installation de lésions irréversibles entraîne une insuffisance rénale chronique lente et progressive dans environ un quart des cas. Le Tableaul montre que $70 \%$ des malades ont une fonction rénale normale après 20 ans d'évolution. L'étude consécutive de 240 reins prélevés au cours d'une transplantation rénale a révélé que $10 \%$ des cas d'insuffisance rénale terminale étaient liés à la maladie de Berger. Dans quelques cas, une disparition prolongée de tous les signes est observée, mais la persistance des dépôts d'Ig A est presque constante dans tous les cas où une seconde biopsie a été faite. Cette persistance des dépôts durant des décennies rend plausible le rôle de facteurs génétiques à leur origine, sans cependant préjuger du rôle de facteurs liés à l'environnement. Après transplantation, les dépôts mésangiaux d'IgA récidivent au niveau du rein transplanté dans environ un tiers des cas [9]. Les conséquences de la récidive sont modestes, sans manifestations cliniques le plus souvent, parfois accompagnées d'une protéinurie minime ou d'une hématurie microscopique. Inversement, la transplantation exceptionnelle de reins de cadavre ayant des dépôts mésangiaux d'IgA (non suspectés car asymptomatiques) chez deux malades atteints d'insuffisance rénale due à une autre cause, a été suivie de la disparition des dépôts.

\section{Facteurs génétiques}

Le rôle des facteurs génétiques dans la maladie de Berger [10] est suggéré - indirectement - par trois ordres d'observations: la distribution géographique, l'existence de formes familiales et l'association à certains marqueurs allotypiques. Premièrement, la distribution géographique de la maladie n'est pas homogène, bien qu'elle soit largement répandue. Elle est évaluée à 2,8/10 000 dans une population de 400000 habitants de la Bretagne du Nord [11]. Sa fréquence par rapport au nombre total de biopsies est de $10 \%$ en Amérique du Nord, de $20 \%$ en Europe et de $40 \%$ au Japon et à Singapour. La 


\begin{tabular}{|c|c|c|c|}
\hline \multicolumn{4}{|c|}{$\begin{array}{c}\text { Tableau I } \\
\text { ÉVOLUTION A LONG TERME }\end{array}$} \\
\hline & $\begin{array}{c}\text { Nombre } \\
\text { de malades } \\
(n)\end{array}$ & $\begin{array}{l}\text { Survie rénale } \\
\text { à } 10 \text { ans } \\
\text { (\%) }\end{array}$ & $\begin{array}{l}\text { Survie rénale } \\
\text { à } 20 \text { ans } \\
\text { (\%) }\end{array}$ \\
\hline $\begin{array}{l}\text { Droz et al. } \\
\text { (1984) [5] }\end{array}$ & 260 & 85 & 75 \\
\hline $\begin{array}{l}\text { D’Amico } \\
\text { (1985) [6] }\end{array}$ & 365 & 85 & 66 \\
\hline $\begin{array}{l}\text { Nicholls et al. } \\
\text { (1984) [7] }\end{array}$ & 244 & 87,5 & - \\
\hline $\begin{array}{l}\text { Beukhof et al. } \\
\text { (1986) [8] }\end{array}$ & 75 & 82 & 73 \\
\hline
\end{tabular}

fréquence de la maladie dans certains groupes ethniques est supérieure à celles des Caucasiens*. Dans l'État de New Mexico par exemple, la fréquence de la maladie est huit fois plus grande chez les Indiens américains comparés aux Anglo-Américains. A l'inverse, la fréquence chez les Noirs américains est plus faible d'un facteur quatre par rapport à cette même population anglo-américaine. Deuxièmement, il existe de nombreux cas de maladies de Berger familiales. Elles ont été observées entre parents et enfants dans une même fratrie ou chez des jumeaux. Une étude collaborative française est en cours $^{* *}$ et a répertorié 23 familles comportant deux membres atteints. Dans 13 autres familles, les deux membres sont respectivement atteints de maladie de Berger et de purpura rhumatoïde. Cette étude devrait permettre de préciser si ces cas ne sont pas seulement dus au hasard, mais plutôt à des facteurs génétiques. Il existe en particulier un excès d'haplotypes HLA identiques chez les germains atteints. Enfin, l'argument le plus convaincant quant au rôle de facteurs génétiques provient du Kentucky, où l'étude d'une trentaine de malades a révélé des ancêtres communs [12]. Ce type d'étude génétique rencontre deux types de difficultés. D'une part, il existe des formes totalement asymptomatiques. A Singa-

\footnotetext{
* Blancs d'origine européenne.

** M. Léry, Inserm U. 155, château de Longchamp, Bois de Boulogne, 75016 Paris.

$\mathrm{m} / \mathrm{s} n^{\circ} 5$ vol. 5 , mai 89
}

leur récidive après transplantation conduit à considérer qu'une anomalie de la réponse immune $\operatorname{IgA}$ existe chez ces malades [15]. Nous verrons que, malgré son caractère défini et apparemment simple, l'événement très probablement initial qu'est le dépôt d'IgA reste une énigme. Notamment, les rôles respectifs de la stimulation par un antigène extrinsèque ou d'une anomalie intrinsèque de l'appareil immunitaire ne sont pas clairs. Plus encore, l'origine même, médullaire ou muqueuse, des IgA présentes dans le mésangium n'est pas indiscutable.

La nature des dépôts mésangiaux d'IgA a été précisée par l'étude directe du tissu rénal à l'aide d'anticorps monoclonaux : ils sont constitués de façon très prédominante sinon exclusive, par des IgA de sousclasse IgAl. Dans un tiers des cas, des IgG sont présentes, elles sont alors de sous-classes IgGl et IgG3 quasi exclusivement $[3,16]$. Cetie restriction de sous-classes rappelle celle qui est observée habituellement au cours des réponses antivirales et est très différente de celle observée dans d'autres glomérulonéphrites de type auto-immun (glomérulonéphrite extramembraneuse et par auto-anticorps anti-membrane basale glomérulaire), où les $\operatorname{IgG}$ déposées sont essentiellement de sous-classes IgGl et IgG4 [17]. L'élution, à partir de fragments obtenus par biopsie à l'aiguille, des IgA mésangiales a permis de préciser que leur structure était variable, dimérique de façon prédominante $(60 \%)$, mais également monomérique (40\%) [18]. Contrairement à la diversité de leur poids moléculaire, la charge des $\operatorname{Ig}$ A éluées s'est révélée être remarquablement homogène et anionique [18]. Comme l'ont montré certains modèles expérimentaux animaux, la charge restreinte et négative de l'IgA pourrait expliquer en partie son affinité pour le mésangium.

L'étude des IgA sériques s'est concentrée, au début des années 1980, sur l'hypothèse selon laquelle les dépôts d'Ig A seraient des complexes immuns circulants se localisant dans le mésangium. Celle-ci était fondée sur l'analogie avec les glomérulonéphrites expérimentales, où l'injection de complexes immuns préformés de grande taille est suivie de leur 
RÉFÉRENCES

13. Wyatt RJ, Kanayama Y, Julian BA, et al. Complement activation in IgA nephropathy. Kidney Int 1987 ; 31 : 1019-23.

14. Waxman FJ, Hebert LA, Cosio FG, et al. Differential binding of immunoglobulin A and immunoglobulin $\mathrm{G}$ immune complexes to primate erythrocytes in vivo. J Clin Invest $1986 ; 77: 82-9$.

15. Feehally J. Immune mechanisms in glomerular IgA deposition. Nephrol Dial Transplant $1988 ; 3: 361-78$.

16. Russell MW, Mestecky J, Julian BA, Galla JH. IgA-associated renal diseases : antibodies to environmental antigens in sera and deposition of immunoglobulins and antigens in glomeruli. J Clin Immunol 1986; 6 : 7486.

17. Noè1 LH, Aucouturier $P$, Monteiro RC Preud'homme JL, Lesavre P. Glomerular and serum immunoglobulin $G$ subclasses in membranous and anti-glomerular basement membrane nephritis. Clin Immunol Immunopathol 1988 ; 46 : $1-9$

18. Monteiro RC, Halbwachs-Mecarelli L, Roque-Barreira MC, Noël LH, Berger J, Lesavre P. Charge and size of mesangial $\operatorname{IgA}$ in IgA nephropathy. Kidney Int $1985 ; 28$ : 666-71.

19. Lesavre P, Digeon M, Bach JF. Analysis of circulating IgA and detection of immune complexes in primary Ig A nephropathy. Clin Exp Immunol 1982 ; 48 : 61-9.

20. Valentijn RM, Radl J, Haajman JJ, et al. Circulating and mesangial secretory component-binding IgA-l in primary $\operatorname{IgA}$ nephropathy. Kidney Int $1984 ; 26$ : 760-6.

21. Monteiro RC, Chevailler A, Noèl LH, Lesavre P. Serum IgA preferentially binds to cationic polypeptides in $\operatorname{Ig} A$ nephropathy. Clin Exp Immunol 1988 ; 73 : 300-6.

22. Delacroix DL, Vaerman JP. L'immunoglobuline A. medecine/sciences $1985 ; 1$ : 348-9.

23. Rambaud JC, Halphen $\mathbf{M}$, Lemaire $\mathbf{M}$. L'immunité humorale intestinale. medecine/sciences $1985 ; 1$ : 350-7.

24. Alley CD, Nash GS, MacDermott RP. Marked in vitro spontaneous secretion of $\operatorname{Ig} A$ by human rid bone marrow mononuclear cells. J Immunol 1982 ; 128 : 2604-8.

25. Mestecky J, Russell MW, Jackson S, Brown TA. The human $\operatorname{IgA}$ system : a reassessment. Clin Immunol Immunopathol $1986 ; 40: 105-14$. localisation mésangiale. Les recherches de «complexes immuns» contenant des $\operatorname{IgA}$ ont montré qu'il existe, chez environ la moitié des malades, des $\operatorname{Ig}$ A circulantes sous forme macromoléculaire [19, 20]. En réalité, ce fait ne préjuge pas de leur réelle nature de complexe antigèneanticorps, puisque l'antigène éventuel n'a pas été identifié. De plus, aucun argument ne permet de considérer que ces $\operatorname{IgA}$ macro-moléculaires jouent un rôle direct dans leur dépôt mésangial. Cette particularité qu'ont les IgA de former des agrégats pourrait être liée à la formation de complexes immuns contenant un antigène exogène, mais également à d'autres facteurs, tels qu'une structure physicochimique particulière favorisant les interactions des domaines Fc, ou une activité autoanticorps anti-IgG. Nous avons dans ce domaine récemment montré l'existence d'IgA circulantes de charge anionique ainsi que la présence, dans le sérum des malades, d'IgA à activité auto-anticorps antiIgG (facteur rhumatoïde IgA). Ces deux anomalies ne sont pas corrélées entre elles, mais l'une et l'autre sont étroitement associées à l'existence d'une récidive des dépôts qui n'apparaît que chez un tiers des malades transplantés [21]. Ce résultat suggère que les $\operatorname{Ig} \mathrm{A}$ anioniques et les $\operatorname{Ig} \mathrm{A}$ à activité facteur rhumatoïde, bien qu'indépendantes, pourraient jouer un rôle dans la formation des dépôts mésangiaux. Par ailleurs, des taux normaux d'IgA anionique et de facteur rhumatoïde IgA ont été trouvés chez les malades atteints de purpura rhumatoïde, qui semble donc faire intervenir des mécanismes différents de ceux impliqués dans la maladie de Berger [21].

La question de l'origine des IgA mésangiales est essentielle, mais n'a pas reçu de réponse définitive. Cette difficulté reflète les incertitudes qui persistent quant à l'origine et à l'importance fonctionnelle relative des monomères et des polymères des deux sous-classes d'IgA chez l'homme sain [22]. Les IgA sont produites par deux systèmes en grande partie fonctionnellement indépendants (figure 3). Les plasmocytes responsables de la production de l'IgA des sécrétions externes [23] sont situés sous les surfaces épithéliales au sein de la lamina propria des muqueuses et des glandes à sécrétion externe. Le transport actif de ces $\operatorname{Ig} \mathrm{A}$ sécrétoires repose sur leur affinité pour le composant sécrétoire épithélial. Les précurseurs B de ces plasmocytes sont stimulés dans les plaques de Peyer de l'intestin ou dans leur équivalent respiratoire. Après stimulation par les antigènes de l'environnement, les précurseurs B s'engagent dans la production IgA de sous-classes $\mathrm{IgAl}$ $(60 \%)$ et IgA2 (40\%), de structure polymérique $(>90 \%)$. Par ailleurs, ces cellules $B$ dérivées des plaques de Peyer quittent le sang périphérique et se localisent dans la lamina propria des muqueuses. Ainsi, une immunisation locale (digestive, par exemple) induit une réponse anticorps IgA sécrétoire dans des tissus éloignés qui n'ont pas été directement stimulés par l'antigène. Inversement, l'IgA sérique est principalement monomérique $(>85 \%)$ et de sous-classe essentiellement IgAl (90\%). L'IgA sérique est produite majoritairement par des plasmocytes de la moelle osseuse [24], en quantité analogue à celle de l'IgG. Seuls l'homme et les primates possèdent des taux élevés de monomère circulant sans que l'avantage en soit connu. L'IgA plasmatique d'origine médullaire pourrait jouer le rôle de seconde barrière éliminant les antigènes ayant pénétré dans l'organisme, par voie digestive notamment, sans entraîner de conséquences inflammatoires. Chez l'homme, l'indépendance des deux systèmes est attestée par le fait que les IgA sériques (monomères ou polymères) ne sont pas transportées vers les lumières et que les IgA polymériques produites par les plasmocytes sousépithéliaux ne contribuent pratiquement pas au pool circulant des IgA. L'absence de corrélation de l'activité anticorps des IgA entre les deux systèmes confirme cette indépendance. Celle-ci n'est toutefois pas absolue, puisque l'immunisation par voie générale peut entraîner, chez des sujets préalablement immunisés par voie muqueuse par le même antigène, une réponse $\operatorname{Ig} \mathrm{A}$ sécrétoire [25]. Inversement, dans certains modèles expérimentaux chez la souris, une immunisation parentérale préalable entraîne une suppression de la réponse sécrétoire à une immunisa- 


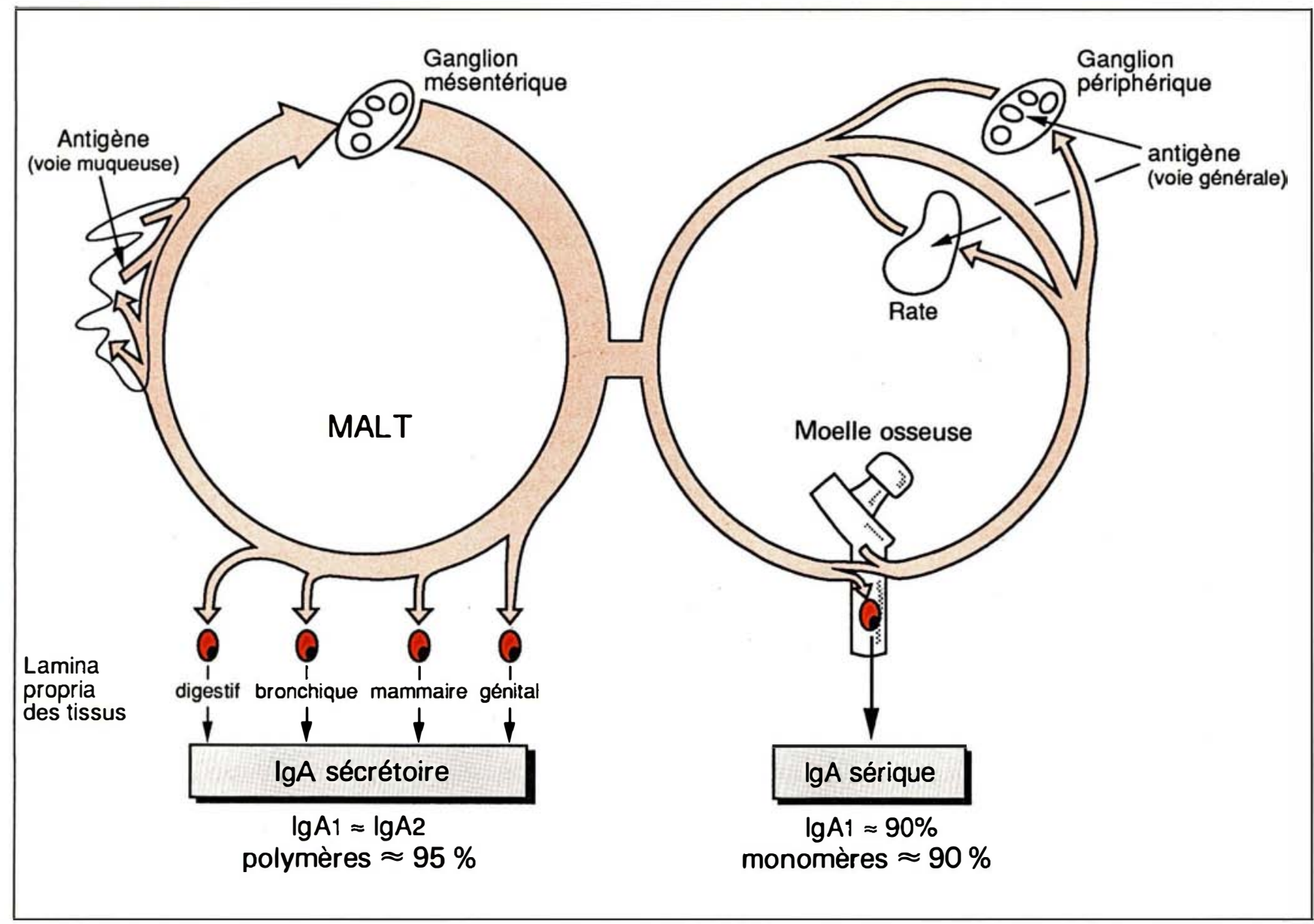

Figure 3. Représentation schématique de la circulation des cellules précurseurs des plasmocytes à IgA. ॥ existe, chez l'homme, deux systèmes fonctionnellement en grande partie distincts (voir texte): le système IgA associé aux muqueuses (MALT, mucosa associated lymphoid tissue) et le système médullaire, produisant respectivement les lgA sécrétoires et les $\lg A$ sériques.

tion par voie muqueuse. Enfin, si une stimulation antigénique muqueuse conduit essentiellement à la localisation des lymphocytes B activés dans les tissus muqueux, une minorité d'entre eux se localise dans la moelle osseuse lorsque la stimulation est répétée. Toutes les particularités du système IgA chez l'homme rendent difficile l'extrapolation des modèles expérimentaux à l'homme [26].

Dans la maladie de Berger, le taux sérique des IgA est élevé dans plus de la moitié des cas (les taux d'IgG et d'IgM sont normaux). Cela est dû à une élévation du taux d'IgAl monomérique circulante [27]. Le taux d'IgA2 et le pourcentage d'IgA polymériques sont comparables aux témoins [19, 27, 28]. Ainsi, il semble qu'existe, au cours de la maladie de Berger, une hyperactivité du système $\operatorname{Ig} \mathrm{A}$ dont témoignent une réponse $\mathrm{m} / \mathrm{s} n^{\circ} 5$ vol. 5 , mai 89
IgA accrue aux stimulations antigéniques systémique et orale [29] et une commutation de classe (ou switch) préférentielle vers les IgAl. Cela a été confirmé directement par l'observation d'une élévation de la synthèse des IgAl par les plasmocytes médullaires des malades [30], alors que la synthèse d'IgA2 est normale; de plus, il existe une élévation du pourcentage d'Ig Al dans le tissu amygdalien [31]. Cette commutation préférentielle vers les $\mathrm{IgAl}$ est donc observée dans les deux compartiments du système IgA. Elle pourrait être en partie liée à des facteurs génétiques, puisque nous avons observé, en collaboration avec G. Keyeux, M.P. Lefranc et G. Lefranc (laboratoire d'immunogénétique, USTL, Montpellier) un polymorphisme des fragments de restrictions des gènes des «régions de switch» $\alpha$ l et $\alpha 2^{*}$ (note p. 292) beaucoup plus impor- tant dans l'ADN des malades, chez qui existent des allèles nouveaux absents chez les sujets normaux.

Le contrôle cellulaire de la synthèse des $\operatorname{IgA}$, au cours de la maladie de Berger, a été largement étudié pour tenter de préciser si l'hyperactivité du système $\operatorname{IgA}$ était liée à un défaut cellulaire, lymphocytaire B ou $\mathrm{T}$ notamment. Les lymphocytes du sang périphérique, stimulés ou non par un mitogène (pokeweed mitogen, PWM), produisent chez le sujet normal des IgA majoritairement polymériques et de sous-classes Al et A2. Il s'agit donc de lymphocytes fonctionnellement proches du système muqueux. Chez les malades, cette réponse est normale dans la majorité des études [32]. Les résultats concernant les fonctions des lymphocytes $T$ auxiliaires ou suppresseurs de la réponse $\operatorname{IgA}$ sont contradictoires. Nous nous sommes attachés, avec 


\section{RÉFÉRENCES}

26. Rifai A. Experimental models for $\operatorname{IgA-}$ associated nephritis. Kidney Int 1987; 31 : 1-7.

27. Van den Wall Bake AWL, Daha MR, Van Der Ark A, Hiemstra PS, Radl J, Van Es LA Serum levels and in vitro production of $\lg A$ subclasses in patients with primary IgA nephropathy. Clin Exp Immunol 1988; 74 : $115-20$

28. Delacroix DL, Elkon KB, Geubel AP, Hodgson HF, Dive C, Vaerman JP. Changes and size, subclass and metabolic properties of serum immunoglobulin $\mathrm{A}$ in liver diseases and in other diseases with high serum immunoglobulin A. J Clin Invest 1983 ; 71 : 358-67.

29. Leinikki PO, Mustonen J, Pasternack A. Immune response to oral polio vaccine in patients with IgA nephropathy. Clin Exp Immunol $1987 ; 68: 33-8$.

30. Van den Wall Bake AWL, Daha MR, Radl J, et al. The bone marrow as production site of the IgA deposited in the kidneys of patients with IgA nephropathy. Clin Exp Immunol $1988 ; 72: 321-5$.

31. Nagy J, Brandtzaeg P. Tonsillar distribution of $\operatorname{IgA}$ and $\operatorname{IgG}$ immunocytes and production of $\operatorname{IgA}$ subclasses and $J$ chain in Tonsillitis vary with the presence or absence of IgA nephropathy. Scand J Immunol 1988 27 : 393-9.

32. Rothschild E, Chatenoud L. T cell subset modulation of immunoglobulin production in IgA nephropathy and membranous glomerulonephritis. Kidney Int 1984 ; 25 : 557-64.

33. Adachi M, Yodoi J, Masuda T, Takatsuki $\mathrm{K}$, Uchino $\mathrm{H}$. Altered expression of lymphocyte $F c \alpha$ receptor in selective $\operatorname{IgA} \operatorname{defi}$ ciency and IgA nephropathy. J Immunol $1983 ; 131: 1246-52$

34. Chevailler A, Monteiro RC, Daëron M Lesavre P. Induction of Fc receptors for $\operatorname{IgA}$ on murine $T$ cell hybridoma by human monoclonal $\operatorname{IgA}$ and by high molecular weight $\operatorname{IgA}$ in $\operatorname{Ig} A$ nephropathy. Clin Exp Immunol 1987 ; 67 : 114-23.

35. Sautès C, Fridman WH. Des lymphokines régulatrices de la production des classes d'anticorps. médecine/sciences 1987; 3: 143-9.

\section{Remerciements}

Nous remercions P. Nusbaum pour son excellente contribution technique.
A. Chevailler en collaboration avec M. Daëron (Inserm U. 255), à l'étude des lymphocytes $T$ porteurs d'un récepteur pour le fragment $\mathrm{Fc}$ des $\operatorname{IgA}(\mathrm{RF}(\alpha)$. L'expression de ce récepteur est augmentée au cours de la maladie de Berger [33]. Les IgA polymériques présentes dans le sérum des malades atteints de néphropathie à IgA induisent puissamment l'expression des récepteurs $F_{c} \alpha$ d'une lignée lymphocytaire $\mathrm{T}$ murine (T2D4). Cette induction est observée avec des concentrations faibles d'IgA sérique de malades, alors que des IgA normales sont sans effet inducteur, même à une concentration cinq fois plus élevée. Ces résultats suggèrent l'existence d'une anomalie qualitative des IgA circulantes au cours de la maladie de Berger [34]. On peut penser que cette IgA anormale, en induisant l'expression du RFca des lymphocytes $T$, entraîne aussi la synthèse d'IgA-binding factor (IgA-BF) qui pourrait jouer un rôle dans l'anomalie de régulation de la synthèse des $\operatorname{Ig} \mathrm{A}$, comme cela a été démontré dans d'autres situations [35].

En conclusion, par sa fréquence et sa gravité la maladie de Berger constitue une préoccupation majeure, d'autant qu'il n'existe pas actuellement de traitement curatif. Les mécanismes du dépôt mésangial des IgA sont très discutés. Deux orientations de recherche nous semblent prometteuses. La première est l'étude de la structure des IgA. Certaines de leurs anomalies pourraient expliquer leur propension à s'agréger et à se déposer dans le mésangium. La seconde orientation est l'étude du trouble de la régulation de la synthèse des $\operatorname{IgA}$ et de la commutation préférentielle de la sous-classe IgAl, observés chez une majorité de malades. Ces anomalies cellulaires jouent probablement un rôle important puisqu'elles conduisent à une synthèse d'IgAl, qui est l'immunoglobuline présente dans les dépôts

\section{Summary}

IgA nephropathy (Berger's disease)

IgA nephropathy has been first described 20 years ago by Berger. This glomerular disease is characterized by the predominant mesangial deposition of IgA and is now recognized as the commonest form of glomerulonephritis in many countries. The disease can have a progressive course leading, over variable periods, to end-stage renal failure. $\operatorname{Ig} A$ nephropathy accounts for about 25 per cent of all primary glomerular diseases and for 10 per cent of the cases of end-stage renal failure. IgA mesangial nephropathy recurs very frequently after transplantation. The pathogenesis of the disease is largely unknown. Genetic factors are likely to play a role as evidenced by the occurrence of familial cases, a higher prevalence among white patients compared to blacks, and the association with major histocompatibility antigens (HLAB35, HLA-DR4). New insights in the mechanisms and functions of the IgA immune system has permitted real progress in the understanding of the immune mechanis m s underlying Ig A nephropathy : two major lines of investigation will potentially lead to a better understanding of the disease and in twin to therapeutic approaches which are missing today. First, the structural studies of IgA are of importance since abnormal molecular weight, charge and ability to interact with complement or cells, may explain the propensity of IgA to aggregate and/or to deposit in the mesangium. Second an impaired regulation of $\operatorname{IgA}$ synthesis and of IgAl-IgA2 switch is likely to play a role : indeed, mesangial deposits contain almost exclusively $\operatorname{IgAl}$ and serum $\mathrm{IgAl}$ is increased in about half of the patients.

\section{TRÉS A PART}

Ph. Lesavre.
* Voir m/s, Lexique Immunologie, suppl. au $n^{\circ} 1$, vol. 5 , p. 5 . 